PROCEEDINGS OF THE

AMERICAN MATHEMATICAL SOCIETY

Volume 127, Number 1, January 1999, Pages 271-278

S $0002-9939(99) 04498-6$

\title{
SHARPER CHANGES IN TOPOLOGIES
}

\author{
GREG HJORTH
}

(Communicated by Andreas R. Blass)

\begin{abstract}
Let $G$ be a Polish group, $\tau$ a Polish topology on a space $X, G$ acting continuously on $(X, \tau)$, with $B \subset X G$-invariant and in the Borel algebra generated by $\tau$. Then there is a larger Polish topology $\tau^{*} \supset \tau$ on $X$ so that $B$ is open with respect to $\tau^{*}, G$ still acts continuously on $\left(X, \tau^{*}\right)$, and $\tau^{*}$ has a basis consisting of sets that are of the same Borel rank as $B$ relative to $\tau$.
\end{abstract}

\section{The PREFACE}

0.1. Theorem (classical). Let $(X, \tau)$ be a Polish space. Let $B \subset X$ be Borel with respect to $\tau$. Then there is a richer topology $\tau^{*} \supset \tau$ such that

(i) every $\tau^{*}$-open set is Borel in $(X, \tau)$;

(ii) $\left(X, \tau^{*}\right)$ is still a Polish space;

(iii) $B$ is open with respect to $\tau^{*}$. (See 13.1 of [3].)

Thus we have that from the point of view of properties that can be stated solely with reference to Borel structure, every theorem provable for open sets holds as well for arbitrary Borel sets. For instance, as shown in [3], we obtain a fast proof that every uncountable Borel set has size $2^{\aleph_{0}}$.

It turns out that this classical theorem can also be proved in the dynamical context.

0.2. Theorem (Sami). Let $S_{\infty}$ be the permutation group on a countably infinite set, viewed as a topological group with the topology of pointwise convergence. Suppose $S_{\infty}$ acts continuously on a Polish space $(X, \tau)$, with an action of the "logic type", and $B \subset X$ is Borel with respect to $\tau$ and $S_{\infty}$-invariant. Then there is a richer topology $\tau^{*} \supset \tau$ such that

(i) every $\tau^{*}$-open set is Borel in $(X, \tau)$;

(ii) $\left(X, \tau^{*}\right)$ is still a Polish space;

(iii) $B$ is open with respect to $\tau^{*}$;

(iv) $S_{\infty}$ acts continuously on $\left(X, \tau^{*}\right)$. (See 4.2 of [5].)

Then later:

0.3. Theorem (Becker-Kechris). Let $G$ be a Polish group. Suppose $G$ acts continuously on a Polish space $(X, \tau)$ and that $B \subset X$ is Borel with respect to $\tau$ and $G$-invariant. Then there is a richer topology $\tau^{*} \supset \tau$ such that

Received by the editors October 17, 1996 and, in revised form, May 13, 1997.

1991 Mathematics Subject Classification. Primary 04A15.

Key words and phrases. Polish group, topological group, topology.

(C)1999 American Mathematical Society 
(i) every $\tau^{*}$-open set is Borel in $(X, \tau)$;

(ii) $\left(X, \tau^{*}\right)$ is still a Polish space;

(iii) $B$ is open with respect to $\tau^{*}$;

(iv) $G$ acts continuously on $\left(X, \tau^{*}\right)$. (See 5.1 .8 of [1].)

Thus it may seem that a happy story has come to a pleasing conclusion.

Yet there remained a gap in our understanding of these theorems regarding changes in Polish topologies. Whereas with 0.1 and 0.2 it was shown that the change could be effected with the minimum possible disturbance to $\tau$, with 0.3 we had only a crude upper bound for the Borel complexity - with respect to $\tau$ - of the open sets in $\tau^{*}$. Thus the authors of [1] were led to ask whether we can in general choose $\tau^{*}$ so that its basic open sets have approximately the same Borel complexity as $B$.

Here we show this. In particular:

0.4. Theorem. Let $G$ be a Polish group. Suppose $G$ acts continuously on a Polish space $(X, \tau)$ and that $B \subset X$ is $F_{\sigma}$ with respect to $\tau$ and $G$-invariant. Then there is a richer topology $\tau^{*} \supset \tau$ such that

(i) every $\tau^{*}$-open set is $F_{\sigma}$ in $(X, \tau)$;

(ii) $\left(X, \tau^{*}\right)$ is still a Polish space;

(iii) $B$ is open with respect to $\tau^{*}$;

(iv) $G$ acts continuously on $\left(X, \tau^{*}\right)$.

And more generally:

0.5. Theorem. Let $G$ be a Polish group and let $\alpha$ be a countable ordinal. Suppose $G$ acts continuously on a Polish space $(X, \tau)$ and that $B$ is $\sum_{\alpha}^{0}(X, \tau)$ and $G$-invariant. Then there is a richer topology $\tau^{*} \supset \tau$ such that

(i) every $\tau^{*}$-open set is $\underset{\sum_{\alpha}^{0}}{\sim}(X, \tau)$;

(ii) $\left(X, \tau^{*}\right)$ is still a Polish space;

(iii) $B$ is open with respect to $\tau^{*}$;

(iv) $G$ acts continuously on $\left(X, \tau^{*}\right)$.

This answers question 5.1.9 of [1].

An unexpected advantage of the new proof is its brevity.

\section{The LANDSCAPE}

1.1. Definition. A topological group is said to be Polish if it is Polish as a topological space - which is to say that it is separable and it allows a complete compatible metric. If $G$ is a Polish group acting continuously on a Polish space $X$, then I will say that $X$ is a Polish $G$-space.

1.2. Definition. Let $G$ be a Polish group and $X$ a Polish $G$-space. For $U \subset G$ and $B \subset X$ we define the Vaught transforms by the specification that $B^{\Delta U}$ is the set of $x \in X$ such that $\{g \in G: g \cdot x \in B\}$ is non-meager and that $B^{* U}$ is the set of $x \in X$ such that $\{g \in G: g \cdot x \in B\}$ is co-meager.

1.3. Lemma (Vaught). Let $\left(A_{i}\right)$ be a sequence of Borel sets, and let $\mathcal{B}$ be a basis of $G$. Fix $U \subset G$ open, $U \neq \emptyset$. Then

$$
\left(\bigcup_{i \in \mathbb{N}} A_{i}\right)^{\Delta U}=\bigcup\left\{A_{i}^{* V} \mid i \in \mathbb{N}, V \subset U, V \neq \emptyset, V \in \mathcal{B}\right\} .
$$

(See [6], [7], or 16.4 of [3].) 
As a word to notation, if $X$ is a set and $\tau$ is a topology on $X$, I will try to use $X$ to denote the topological space - that is, $X$ equipped with the topology - as long as the intention is clear. When more than one topology is being considered on $X$, we will need to be specific, and instead use $(X, \tau)$, or $\left(X, \tau^{*}\right)$, and so on.

1.4. Definition. If $G$ is a group and $d$ is a metric on $G$, then $d$ is said to be right invariant if for all $g, h_{1}, h_{2} \in G$

$$
d\left(h_{1} \cdot g, h_{2} \cdot g\right)=d\left(h_{1}, h_{2}\right) .
$$

One can similarly define the notion of left invariant metric. Note that these are companion notions, since if $d$ is left invariant, then $d^{*}$ defined by $d^{*}(g, h)=$ $d\left(g^{-1}, h^{-1}\right)$ will be right invariant.

1.5. Theorem (Birkhoff-Kakutani). Any Polish group has a compatible right invariant metric. (See [2], 8.3.)

The metric provided by 1.5 will be compatible with the topology, but not necessarily complete.

1.6. Corollary. Any Polish group has a compatible right invariant metric bounded by 1 - that is $\forall g, h \in G d(g, h) \leq 1$.

Proof. Given $d$ as in 1.5, let $d^{*}(g, h)=d(g, h) /[1+d(g, h)]$.

1.7. Definition. Let $Y$ be a topological space and let $d$ be a compatible metric bounded by 1 . Then let $\mathcal{L}(Y, d)$ be the set of (necessarily continuous) functions

$$
f: Y \rightarrow[0,1]
$$

such that for all $y_{1}, y_{2} \in Y$

$$
\left|f\left(y_{1}\right)-f\left(y_{2}\right)\right| \leq d\left(y_{1}, y_{2}\right) .
$$

1.8. Lemma. $\mathcal{L}(Y, d)$ is compact in the topology of pointwise convergence. If $Y$ is separable, then it is also metrizable.

Proof. The first statement is Tychonov's theorem, while the second follows since for $Q=\left(a_{i}\right)_{i \in \mathbb{N}} \subset Y$ dense and countable we can identify $\mathcal{L}(Y, d)$ with a closed subset of $[0,1]^{Q}$; this last space has a metric given by

$$
d(\vec{x}, \vec{y})=\sum\left\{2^{-i} \cdot\left|x\left(a_{i}\right)-y\left(a_{i}\right)\right|: i \in \mathbb{N}\right\} .
$$

1.9. Definition. Let $G$ be a Polish group and let $d$ be a right invariant compatible metric on $G$ that is bounded by 1 . Then for $f \in \mathcal{L}(G, d)$ let $g \cdot f$ be defined by $(g \cdot f)(h)=f(h g)$ for all $h \in G$.

1.10. Lemma. This defines an action on $\mathcal{L}(G, d)$ under which it becomes a compact Polish G-space.

Proof. By right invariance of $d$, along with the observation that

$$
\left(g_{1} g_{2} \cdot f\right)(h)=f\left(h g_{1} g_{2}\right)=g_{2} \cdot f\left(h g_{1}\right)=\left(g_{1} \cdot\left(g_{2} \cdot f\right)\right)(h),
$$

this defines an action of $G$ on $\mathcal{L}(G, d)$.

Continuity follows by the definition of the space and its topology. If we fix $h \in G$, and let $U$ be an open neighborhood of $h$ such that for all $h^{\prime} \in U\left(d\left(h, h^{\prime}\right)<\epsilon\right)$, then 
for $g \in G$ small enough - say, $g \in W$ where $W$ is an open neighbourhood of the identity - to ensure that $h g \in U$, and all $f \in \mathcal{L}(G, d)$

$$
|g \cdot f(h)-f(h)|=_{d f}|f(h g)-f(h)| \leq d(h g, h)<\epsilon .
$$

Thus in particular if $V$ is the open neighbourhood of some $f_{0} \in \mathcal{L}(G, d)$ consisting of $f \in \mathcal{L}(G, d)$ such that $\left|f(h)-f_{0}(h)\right|<\epsilon$, and $V_{0}$ is the open neighbourhood consisting of $f \in \mathcal{L}(G, d)$ such that $\left|f(h)-f_{0}(h)\right|<2 \epsilon$, then we will have for all $g \in W, f \in V$ that $g \cdot f \in V_{0}$.

Compactness is 1.8 .

For the sake of being thorough:

1.11. Definition. Let $(X, \tau)$ be a Polish space (with $\tau$ the Polish topology). Then the $\sum_{1}^{0}(X, \tau)$ sets are the open sets in $(X, \tau)$; for $\alpha$ a countable ordinal, a set is

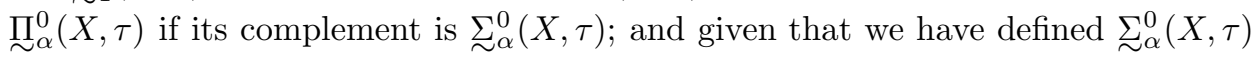
all $\alpha<\delta$, we say that a set is $\sum_{\delta}^{0}(X, \tau)$ if it has the form $\bigcup\left\{A_{i}: i \in \mathbb{N}\right\}$ where each $A_{i}$ is $\underset{\sim \alpha(i)}{\prod_{\alpha}^{0}}(X, \tau)$ for some $\alpha(i)<\delta$.

Thus the $\prod_{2}^{0}(X, \tau)$ sets are precisely the $G_{\delta}$ sets in $(X, \tau)$, and the $\sum_{2}^{0}(X, \tau)$ are the $F_{\sigma}$ sets. Note by 1.3 and a transfinite induction, if $(X, \tau)$ is a Polish $G$ space, $B \in \sum_{\sim}^{0}(X, \tau)$ (or $\left.\prod_{\alpha}^{0}(X, \tau)\right), U \subset G$ open, then $B^{\Delta U}$ (respectively $B^{* U}$ ) is $\sum_{\alpha}^{0}(X, \tau)$ (or $\prod_{\sim}^{0}(X, \tau)$ respectively). The base step of this induction begins with the observation that if $B$ is open, then $B^{\Delta U}=\{x \in X: \exists g \in U(g \cdot x \in B)\}$, and so is open.

1.12. Lemma (classical). Let $(X, \tau)$ be a Polish space and let $X_{0}$ be $\prod_{2}^{0}(X, \tau)$. Then $X_{0}$ is Polish in the relative topology. (See 3.11 of [3].)

1.13. Lemma (classical). Let $\left(X_{i}\right)_{i \in \mathbb{N}}$ be a sequence of Polish spaces; then $\prod\left\{X_{i}: i \in \mathbb{N}\right\}$ in the product topology is Polish. (See 3.3 of [3], or use the proof of the second half of 1.8.)

1.14. Lemma (classical). Let $\left(\tau_{i}\right)_{i \in \mathbb{N}}$ be an increasing sequence of Polish topologies on $X$. Then the topology generated by the union $\bigcup\left\{\tau_{i}: i \in \mathbb{N}\right\}$ is Polish. (See 3.3 of $[3]$ or $[5]$.)

1.15. Definition. Let $G$ be a Polish group, $X$ and $Y$ Polish $G$-spaces. $\pi: X \rightarrow Y$ is a $G$-mapping if for all $g \in G, x \in X$

$$
\pi(g \cdot x)=g \cdot \pi(x)
$$

it is a continuous (or Borel) G-mapping if it is also continuous (respectively, the pullback of open sets are Borel); it is a G-embedding if it is one-to-one.

1.16. Definition. Let $d$ be a right invariant metric on $G$ and $\mathcal{O} \subset X$ open. Then $\varphi^{\mathcal{O}}: X \rightarrow \mathcal{L}(G, d)$ is the map $x \mapsto \varphi_{x}^{\mathcal{O}}$, where for $h \in G$

$$
\varphi_{x}^{\mathcal{O}}(h)=d(h,\{g \in G: g \cdot x \in \mathcal{O}\})=_{d f} \inf \{d(h, g): g \cdot x \in \mathcal{O}\} .
$$

1.17. Lemma. $\varphi^{\mathcal{O}}$ is a $G$-mapping.

Proof. Fix $\bar{g} \in G$. Then for all $h \in G$

$$
\varphi_{\bar{g} \cdot x}^{\mathcal{O}}(h)=d(h,\{g \in G: g \bar{g} \cdot x \in \mathcal{O}\}),
$$


which by definition equals

$$
\inf \{d(h, g): g \bar{g} \cdot x \in \mathcal{O}\},
$$

which by right invariance equals

as required.

$$
\begin{gathered}
\inf \{d(h \bar{g}, g \bar{g}): g \bar{g} \cdot x \in \mathcal{O}\}=\inf \left\{d\left(h \bar{g}, g^{\prime}\right): g^{\prime} \cdot x \in \mathcal{O}\right\} \\
={ }_{d f} \varphi_{x}^{\mathcal{O}}(h \bar{g})=_{d f}\left(\bar{g} \cdot \varphi_{x}^{\mathcal{O}}\right)(h),
\end{gathered}
$$

Note that for $\mathcal{O}$ open, $\varphi_{x}^{\mathcal{O}}(h)>0$ if and only if there is an open set $V \subset G$ with $h \in V$ and $\forall g \in V(g \cdot x \in X \backslash \mathcal{O})$. Thus if $W$ is open in $G$ and $\mathcal{C}=X \backslash \mathcal{O}$, then $x \in \mathcal{C}^{\Delta W}$ if and only if there is some $h \in W$ with $\varphi_{x}^{\mathcal{O}}(h)>0$. The point here is that this last condition is open in the topology on $\mathcal{L}(G, d)$.

1.18. Notation. For $g$ a point in a metric space $(M, d), q \in \mathbb{Q}^{+}$let $B_{q}^{d}(g)$ be the ball of all points in $M$ with $d(x, g)<q$.

\section{THE PROOF}

The rest will be brief.

2.1. Lemma. Let $G$ be a Polish group, $(X, \tau)$ a Polish $G$-space, $U_{i} \subset G$ open, $F_{i} \subset X$ in $\sum_{2}^{0}(X, \tau)$, each $i \in \mathbb{N}$. Then there is a Polish topology $\tau^{*}$ on $X$ such that

(i) $\left(X, \tau^{*}\right)$ is a Polish $G$-space;

(ii) $\tau \subset \tau^{*}$;

(iii) $\mathcal{O} \in \tau^{*} \Rightarrow \mathcal{O} \in \sum_{\sim}^{0}(X, \tau)$;

(iv) $F_{i}^{\Delta U_{i}} \in \tau^{*}$.

Proof. By 1.3 it suffices to show that if $\left(\mathcal{C}_{i}\right)_{i \in \mathbb{N}}$ is a sequence of closed sets in $(X, \tau)$, then we can find $\tau^{*}$ satisfying (i), (ii), and (iii) above, with $\mathcal{C}_{i}^{\Delta W}$ open for each $W \subset G$. For notational simplicity let us concentrate on achieving this outcome for a single $\tau$-closed set $\mathcal{C}$; the more general case has a similar proof.

So fix $\mathcal{C} \subset X$ to be $\tau$-open, and suppose that $\mathcal{C}=X \backslash \mathcal{O}$. Let $\hat{X} \subset X \times \mathcal{L}(G, d)$ be given by $\hat{X}=\left\{\left(x, \varphi_{x}^{\mathcal{O}}\right): x \in X\right\}$. By 1.10 the $X \times \mathcal{L}(G, d)$ is a Polish $G$-space, or rather, can be naturally viewed as such, by taking the product of the actions; by 1.17 the map

$$
\begin{aligned}
\pi: X & \rightarrow X \times \mathcal{L}(G, d) \\
x & \mapsto\left(x, \varphi_{x}^{\mathcal{O}}\right)
\end{aligned}
$$

is a $G$-embedding. Let $\tau_{0}$ be the product topology on $X \times \mathcal{L}(G, d)$.

Claim 1. $\hat{X}$ is $\underset{\sim}{\prod_{2}^{0}}\left(X \times \mathcal{L}(G, d), \tau_{0}\right)$.

Proof of claim. Let $G_{0}$ be a countable dense subset of $G$. Then to assert that for $x \in X$ and $f \in \mathcal{L}(G, d)$ we have $f=\varphi_{x}^{\mathcal{O}}$ amounts to asserting that

$$
\forall q \in \mathbb{Q} \forall g \in G_{0}\left(f(g)<q \Leftrightarrow \exists h \in B_{q}(g)(h \cdot x \in \mathcal{O})\right) .
$$

For any particular $q$ and $g$, the statement that $f(g)<q \Leftrightarrow \exists h \in B_{q}(g)(h \cdot x \in \mathcal{O})$ is a Boolean combination of $\tau_{0}$-open sets and hence clearly $\prod_{2}^{0}\left(X \times \mathcal{L}(G, d), \tau_{0}\right)$, and must therefore be the countable intersection over $\mathbb{Q}$ and $G_{0}$.

(Claim $\square$ )

So $\hat{X}$ is a Polish $G$-space by 1.12 , and we have a $G$-embedding $\pi: X \rightarrow \hat{X}$, obtained by restricting the map $\pi$ into $X \times \mathcal{L}(G, d)$. Use $\tau_{0}$ to also denote the topology of $X \times \mathcal{L}(G, d)$ restricted to $\hat{X}$. 
Claim 2. If $W \subset \hat{X}$ is $\tau_{0}$-open, then $\pi^{-1}(W)$ is $\sum_{2}^{0}(X, \tau)$.

Proof of claim. Let $G_{0}$ be a countable dense subset of $G$, and let

$$
\mathcal{B}=\left\{B_{q}(\bar{g}): \bar{g} \in G_{0}, q \in \mathbb{Q}^{+}\right\}
$$

be a countable subbasis of $G$. It suffices to prove the claim for a subbasic open set in $\hat{X}$ supported on the $\mathcal{L}(G, d)$ coordinate. But for $x \in X, g \in G$

$$
\varphi_{x}^{\mathcal{O}}(g)>q \Leftrightarrow \exists \bar{q} \in \mathbb{Q}^{+} \exists V \in \mathcal{B}\left(B_{q+\bar{q}}(g) \subset V \wedge \forall h \in V(h \cdot x \in C)\right),
$$

which is $\sum_{2}^{0}(X, \tau)$, while

$$
\varphi_{x}^{\mathcal{O}}(g)<q \Leftrightarrow \exists h \in B_{q}(g)(h \cdot x \in \mathcal{O}),
$$

which is $\tau$-open.

Now let $\tau^{*}$ be the topology consisting of sets of the form $\pi^{-1}(W)$, where $W \subset \hat{X}$ is $\tau_{0}$ open. Since it follows from the definition of $\pi$ that $\pi$ is an open mapping, we have $\tau \subset \tau^{*}$. Since $\pi$ is a $G$-embedding, we obtain that $\left(X, \tau^{*}\right)$ is a Polish $G$-space. Following the remark after $1.17, \mathcal{C}^{\Delta V}$ is open in $\left(X, \tau^{*}\right)$ for any open $V \subset G$.

The more general case, where we consider many $\mathcal{C}_{i}$, follows a similar proof. Now $\hat{X}$ will be a $\prod_{2}^{0}$ subset of $X \times(\mathcal{L}(G, d))^{\mathbb{N}}$, where the $i$ th copy of $\mathcal{L}(G, d)$ is responsible for making sets of the form $\mathcal{C}_{i}^{\Delta V}$ open.

2.2. Theorem. Let $G$ be a Polish group, $(X, \tau)$ a Polish $G$-space, $U_{i} \subset G$ open, $B_{i} \subset X$ in $\sum_{\alpha}^{0}(X, \tau)$, for each $i \in \mathbb{N}$ for $\alpha$ some fixed countable ordinal. Then there is a Polish topology $\tau^{*}$ on $X$ such that

(i) $\left(X, \tau^{*}\right)$ is a Polish $G$-space;

(ii) $\tau \subset \tau^{*}$;

(iii) $\mathcal{O} \in \tau^{*} \Rightarrow \mathcal{O} \in \sum_{\alpha}^{0}(X, \tau)$;

(iv) for each $i, B_{i}^{\Delta U_{i}} \in \tau^{*}$.

Proof. By induction on $\alpha$.

If $\alpha$ is an infinite limit ordinal, then we may assume that each $B_{i}=\bigcup\left\{B_{i, j}\right.$ : $j \in \mathbb{N}\}$, with each $B_{i, j}$ in $\underset{\sim}{\sum_{\alpha(j)}^{0}}(X, \tau)$ some fixed $\alpha(j)<\alpha$. Then for $\mathcal{B}$ a basis for $G$, we can employ our inductive hypothesis to find a sequence of Polish topologies $\left(\tau_{j}\right)_{j \in \mathbb{N}}$ such that

$\left(\mathrm{i}^{\prime}\right)$ each $\left(X, \tau_{j}\right)$ is a Polish $G$-space;

(ii') $\tau \subset \tau_{j}$

(iii') $\mathcal{O} \in \tau_{j} \Rightarrow \mathcal{O} \in \sum_{\alpha(j)}^{0}(X, \tau)$;

$\left(\mathrm{iv}^{\prime}\right)$ for each $i \in \mathbb{N}, W \in \mathcal{B}, B_{i, j}^{\Delta W} \in \tau_{j}$.

Let $Y=\Pi\left\{\left(X, \tau_{j}\right): j \in \mathbb{N}\right\}$. By 1.13 this is a Polish space. Note also that it is in fact a Polish $G$-space in the product action. Let $\tau^{*}$ be the topology generated by taking $\bigcup\left\{\tau_{j}: j \in \mathbb{N}\right\}$ as a subbasis. Then $\left(X, \tau^{*}\right)$ is homeomorphic as a $G$-space to the diagonal $\{\vec{x} \in Y: \forall i(x(i)=x(i+1))\}$, which is a closed invariant subset of $Y$ since each $\tau_{i}$ includes $\tau$. It follows from the definitions that under $\pi: X \rightarrow Y$ defined by $(\pi(x))(i)=x$ we have that the pullbacks of open sets are all $\sum_{\alpha}^{0}(X, \tau)$. Thus, as before, $\tau^{*}$ is as required.

For the inductive step, suppose that $\alpha=\beta+1$ and we have already established the theorem for $\beta$. Then we may assume that each $B_{i}=\bigcup\left\{B_{i, j}: j \in \mathbb{N}\right\}$, each $B_{i, j}$ is $\Pi_{\beta}^{0}(X, \tau)$. Let $\tau^{\prime}$, by the inductive hypothesis, be a topology on $X$ so that:

$\left(\mathrm{i}^{\prime \prime}\right)\left(X, \tau^{\prime}\right)$ is a Polish $G$-space; 
(ii') $\tau \subset \tau^{\prime}$

(iii") $\mathcal{O} \in \tau^{\prime} \Rightarrow \mathcal{O} \in \sum_{\sim}^{0}(X, \tau)$;

(iv') for each $i \in \mathbb{N}, W \in \mathcal{B}, B_{i, j}^{* W} \in \prod_{1}^{0}\left(\tau^{\prime}\right)$.

Thus we can apply 2.1 and find a Polish topology $\tau^{*}$ such that

(i*) $\left(X, \tau^{*}\right)$ is a Polish $G$-space;

(ii*) $\tau \subset \tau^{\prime} \subset \tau^{*}$;

(iii $\left.{ }^{*}\right) \mathcal{O} \in \tau^{*} \Rightarrow \mathcal{O} \in \sum_{2}^{0}\left(X, \tau^{\prime}\right) \subset \sum_{\beta+1}^{0}(X, \tau)$;

$\left(\right.$ iv $\left.^{*}\right)$ for each $i \in \mathbb{N}, W_{0}, W_{1} \in \mathcal{B},\left(B_{i, j}^{* W_{0}}\right) \Delta W_{1} \in \sum_{1}^{0}\left(\tau^{*}\right)$.

Now we observe that for $B_{i}=\bigcup\left\{B_{i, j}: j \in \mathbb{N}\right\}, W \in \mathcal{B}$

$$
B_{i}^{\Delta W}=\bigcup\left\{\left(B_{i, j}^{* W_{0}}\right)^{\Delta W_{1}}: j \in \mathbb{N}, W_{0}, W_{1} \in \mathcal{B}, W_{0} \cdot W_{1} \subset W\right\},
$$

and thus is open with respect to $\tau^{*}$.

2.3. Corollary. Let $G$ be a Polish group, $(X, \tau)$ a Polish $G$-space, $B_{i} \subset X$ a sequence of $G$-invariant sets - that is, $\forall g \in x \in B_{i}\left(g \cdot x \in B_{i}\right)$. Suppose $B_{i} \in$ $\sum_{\alpha}^{0}(X, \tau)$. Then there is a Polish topology $\tau^{*}$ on $X$ such that

(i) $\left(X, \tau^{*}\right)$ is a Polish $G$-space;

(ii) $\tau \subset \tau^{*}$;

(iii) $\mathcal{O} \in \tau^{*} \Rightarrow \mathcal{O} \in \Sigma_{\alpha}^{0}(X, \tau)$;

(iv) each $B_{i} \in \tau^{*}$.

Proof. By 2.2, if $U$ is any non-empty open subset of $G$, we have that each $B_{i}^{\Delta U}=B_{i}$ by invariance.

\section{SOME CONNECTIONS}

The construction above can be given other tasks.

For instance, as remarked by Alexander Kechris, if $\left(\mathcal{O}_{i}\right)_{i \in \mathbb{N}}$ forms a basis for the topology for the Polish $G$-space $(X, \tau)$, then for $d$ a right invariant metric we may consider the function

$$
\begin{gathered}
\rho: X \rightarrow(\mathcal{L}(G, d))^{\mathbb{N}} \\
\rho: x \mapsto\left(\varphi_{x}^{\mathcal{O}_{i}}\right)_{i \in \mathbb{N}} .
\end{gathered}
$$

It follows from the proof of 2.1 that $\rho$ is a $G$-map such that the pullback of any open set is ${\underset{\sim}{\sim}}_{2}^{0}(X, \tau)$ - in other words, $\rho$ is a Baire class 1 function. Since we have chosen enough open sets, $\rho$ is in fact a Baire class $1 G$-embedding from $(X, \tau)$ to a compact Polish $G$-space. Thus we obtain a new proof of a theorem from [1] that for every Polish group there is a universal Polish $G$-space $\mathcal{U}_{G}$, such that every other Polish $G$ space allows a Borel $G$ embedding into $\mathcal{U}_{G}$.

It is known from work of Megrelishvili that in general there may exist Polish groups $G$ for which there is a Polish $G$-space that allows no continuous $G$-embedding into a compact Polish $G$-space. Thus we may choose to view this as a kind of optimal result - one in general cannot hope for a compact Polish $G$-space that is universal via continous maps, but there does exist a compact Polish $G$-space that is universal via Baire class $1 G$-embeddings.

Kechris has also shown that in this construction the pointwise image of $X$ under $\rho$ is $\underset{\sim}{\prod_{2}^{0}}$ in the product topology on $(\mathcal{L}(G, d))^{\mathbb{N}}$, thereby strengthening the sense that this is optimal granted the Megrelishvili counterexample. 
Another application was noted by Ramez Sami, who commented that we hereby obtain a generalization of a result from [5] that was initially proved only for the Polish group $S_{\infty}$.

3.1. Proposition. Let $G$ be a Polish group, $(X, \tau)$ a Polish $G$-space, and suppose that there are less than $2^{\aleph_{0}}$ many orbits. Then any invariant $\prod_{\alpha+1}^{0}(X, \tau)$ contains $a \underset{\sim}{\prod_{\alpha+1}^{0}}(X, \tau)$ orbit.

Proof. Let $B$ be the invariant set. We can find $\tau^{*}$ as in 2.2 so that $\tau \subset \tau^{*}$ and $B \in \underset{\sim}{\sim} \prod_{2}^{0}\left(X, \tau^{*}\right)$ and every $\tau^{*}$-open set is $\sum_{\alpha}^{0}(X, \tau)$. Since $B$ does not contain $2^{\aleph_{0}}$ many orbits, the orbit equivalence relation is not meager, and hence we may find a $\underset{\sim}{\prod_{2}^{0}}\left(X, \tau^{*}\right)$ orbit, as in [5] (or see [1]). Then this orbit will be $\underset{\sim}{\prod_{\alpha+1}^{0}}(X, \tau)$, as required.

Using the proof of 5.1 .10 of [1] one can reduce the assumption that there are less than $2^{\aleph_{0}}$ many orbits to the weaker hypothesis that the orbit equivalence relation does not Borel reduce $E_{0}$, the equivalence relation of eventual agreement on countable sequences of integers.

\section{ACKNOWLEDGEMENTS}

I thank Su Gao and Alexander Kechris, who both read through an earlier draft of this paper and made many helpful suggestions; Kechris in particular caught various errors and alerted me to the Megrelishvili paper.

\section{REFERENCES}

1. H. Becker and A.S. Kechris, The descriptive set theory of Polish group actions, to appear in the London Mathematical Society Lecture Notes Series, 232, 1996. CMP 97:06

2. E. Hewitt and K.A. Ross, Abstract harmonic analysis, Vol. I, Springer-Verlag, Berlin and New-York, 1979. MR 81k:43001

3. A.S. Kechris, Classical descriptive set theory, Graduate Texts in Mathematics Series, SpringerVerlag, Berlin and New-York, 1995. MR 96e:03057

4. M.G. Megrelishvili, A Tikhonov G-space that does not have compact $G$-extension and $G$ linearization, Uspekhi Matematicheskikh Nauk, vol. 43(1988), pp. 145-6. MR 89e:54080

5. R. Sami, Polish group actions and the topological Vaught conjecture, Transactions of the American Mathematical Society, vol. 341(1994), pp. 335-353. MR 94c:03068

6. R. Vaught, A Borel invariantization, Bulletin of the American Mathematical Society, vol. 79(1973), pp. 1291-5. MR 48:10818

7. R. Vaught, Invariant sets in topology and logic, Fundamenta Mathematica, vol. 82(1974), pp. 269-94. MR 51:167

Department of Mathematics, University of California los Angeles, Los Angeles, CALIFORNia 90095-1555

E-mail address: greg@math.ucla.edu 